\title{
ANTROPOLOGÍA
}

\section{EDUCACIÓN MATERNAL:}

Un estudio etnográfico en el consultorio de salud de las Fuentezuelas.

Linares Abad, Manuel *; Moral Guitiérrez, Inés **

Linares Abad, Miguel ***; Sáenz Monzón, Esperanza ***

*Enfermero. Matrón. Antropólogo. Profesor Titular de E. U. de la Universidad de Jaén.

**Enfermera. Hospital Universitario Ciudad de Jaén.

***Matrones. Distrito Sanitario de Jaén.

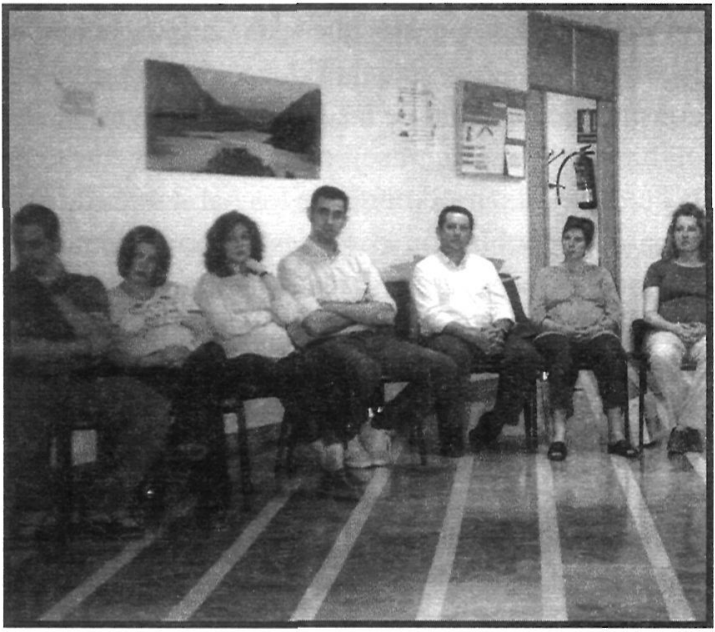

SUMMARY

O tudy qualitative based on the description etnográphic of a program of maternal education developed in the center of health of the Fuentezuelas of Jaén.

Material and method: it is a study of unique case, selecting the program of education maternal study object for a bigger access easiness to the field. were used as technical of collection data the participant observation and the interview in group.

Main results: The program of education maternal study object breaks up with the traditional system of maternal education, it is developed on the base of theoretical sessions and terrestrial and aquatic gymnastic practices. The pregnant ones manifest the improvement of the corporal functionality and the reduction of fears like the most outstanding consequences in the activities of maternal education.

Main conclusions: The theoretical sessions of the program of maternal education give opportunity to the pregnant ones to exchange experiences and increase the solidarity and cohesion of the group.

Words key: Pregnancy, maternal education, maternity, preparation to the childbirth.

\section{RESUMEN}

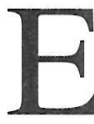
studio cualitativo basado en la descripción etnográfica de un programa de educación maternal desarrollado en el centro de salud de las Fuentezuelas de Jaén.

Material y método: Se trata de un estudio de caso único, seleccionando el programa de educación maternal objeto de estudio por una mayor facilidad de acceso al campo Se utilizaron como técnicas de recogida de datos la observación participante y la entrevista en grupo.

Resultados principales: El programa de educación maternal objeto de estudio rompe con el sistema tradicional de educación maternal, está desarrollado sobre la base de sesiones teóricas y prácticas gimnásticas terrestres y acuáticas.

Conclusiones principales: Las sesiones teóricas del programa de educación maternal le dan oportunidad a las embarazadas para intercambiar experiencias y potenciar la solidaridad y cohesión del grupo.

Palabras clave: Embarazo, educación maternal, maternidad, preparación al parto. 


\section{INTRODUCCIÓN}

El sistema sanitario autónomo andaluz puso en marcha en la década de los años 80 un ambicioso programa de salud Materno-Infantil dentro del marco de la Atención Primaria de Salud. Entre las actividades encargadas de proporcionar estrategias y recursos efectivos a la mujer embarazada se encuentran las actividades de Educación Maternal que "....en consonancia con el ámbito cultural y social donde se asienten y desde el respeto al mismo, se estructuran como un conjunto de actividades que profesionales sanitarios, individuos y comunidad realizan para contribuir a que el embarazo se vivencie de forma positiva y faciliten un parto y puerperio normal en el aspecto físico y emocional" ( Rufo y Fernández, 1987).

La Educación Maternal hunde sus raíces en el método de psicoprofilaxis obstétrica-preparación al parto- de Lamaze, que tuvo su gestación en la Escuela Rusa (Reeder, Martín y Koniak, 1995). El fundamento de este método se basa en el concepto de Paulov de percepción del dolor y su teoría de los reflejos condicionados-sustitución de reflejos condicionados desfavorables por reflejos condicionados favorables-. Consiste por tanto en una preparación mental para el parto. Fue Lamaze quien introdujo en este método la respiración rápida y superficial como técnica de apoyo a lo establecido por la Escuela Rusa. En España, Álvaro Aguirre de Cárcer fue el introductor del método en la década de los años 70 acuñando el nombre de Educación Maternal.

Las actividades de Educación Maternal se erigen como piedra angular de la Educación para la Salud en el área de la mujer, por transmitir conocimientos necesarios que llevan a acciones de autocuidado y por tanto adquisición de responsabilidad en la mejora y mantenimiento de la salud de la mujer durante el periodo de embarazo, parto y puerperio (Sebastía, 2000). Bajo este marco teórico de estrategia de intervención sanitaria para promover un estado de salud óptimo en torno a la mujer embarazada y con la referencia de las funciones de la educación como la de “...preparar a las personas para ganarse la vida y la de ayudarlas a que puedan realizarse personalmente y aporten su contribución a la sociedad" (Horton y Hunt, 1988) en este estudio y con un paradigma cualitativo de referencia, nuestro objetivo fundamental se centra en realizar una descripción etnográfica de las actividades de Educación Maternal que se realizan en nuestro entorno social y conocer de forma detallada la organización, estructura y contenidos de dichas actividades. Este trabajo debe entenderse bajo un contexto no sólo sanitario sino social y cultural y utilizarlo como un elemento que intenta poner de manifiesto, a través de la descripción e interpretación, los cuidados que prestan los profesionales sanitarios en el área de salud de la mujer.

\section{MATERIAL Y MÉTODO}

Se trata éste, de un estudio de caso único basado en criterios, puesto que esta investigación se localiza en el programa de Educación Maternal del consultorio de salud de las Fuentezuelas situado en un barrio de nueva creación en la zona nordeste de la ciudad de Jaén. El perfil de los habitantes de esta zona es de población compuesta por matrimonios jóvenes en edad de procreación que viven en su mayoría en viviendas residenciales con espacios ajardinados, piscina y columpios dedicados al ocio y recreo.

El hecho de seleccionar este centro para realizar la investigación viene justificado por una más fácil accesibilidad al campo, tras tomar contacto inicialmente con la persona responsable de este programa y habiéndole presentado el proyecto de nuestra investigación éste no puso objeción alguna al desarrollo de la misma. En conversaciones informales, unas veces cara a cara y otras por vía telefónica se fijó una fecha para la entrada al campo. Nuestro informante clave nos aconsejó comenzar el trabajo de campo coincidiendo con el inicio de las actividades de educación maternal para un nuevo grupo de mujeres embarazadas. De esta forma minimizábamos el fenómeno de extrañamiento que podía suponer la permanencia en estas actividades de una persona ajena al centro de salud. El grupo de mujeres estaba constituido por 10 embarazadas multíparas -una tercigesta y nueve secundigestas- y 5 embarazadas nulíparas.

El trabajo de campo propiamente dicho se desarrolló desde la primera semana de Marzo de 2002 en que se tomó contacto con el informante clave, matrón responsable del programa, hasta el 20 de mayo de 2002. Durante este periodo hemos 
realizado seis visitas al campo, coincidiendo con diferentes sesiones de educación maternal, cuatro visitas a las sesiones teóricas, una visita a una sesión de ejercicios gimnásticos y una visita a una sesión de ejercicios acuáticos en piscina que nos han permitido conocer el hilo conductor del programa. Bien es verdad que el trabajo en el campo fue discontinuo adaptándonos a la periodicidad de las actividades y reconocemos como un factor a tener en cuenta el no haber podido asistir como observadores a todas las sesiones.

En este artículo presentamos una descripción e interpretación de los datos recogidos solamente en las sesiones teóricas del programa de educación maternal, dado que presentar el trabajo en su totalidad extralimitaría la extensión dedicada a este tipo de artículos.

Las técnicas utilizadas para la recogida de datos han sido la observación participante apoyada en un cuaderno de campo, hemos utilizado cámara fotográfica durante nuestra estancia en el campo como una técnica más de recogida de datos. También se utilizó como técnica de recogida de datos la entrevista en grupo (Ruiz, 1996), dado que la organización de las actividades favorece el uso de la misma. Para todas las sesiones se procuró y se consiguió llegar un tiempo antes para mantener conversaciones con nuestro informante clave que nos permitieran recoger datos para el estudio.

\section{HALLAZGOS Y DISCUSIÓN}

\section{Programa de Educación Maternal en el centro de salud de las Fuentezuelas.}

El programa de educación maternal del centro de las Fuentezuelas nace como respuesta a la demanda de la población emergente, la expansión del nuevo barrio provoca un aumento de afiliados en la zona de salud y por consiguiente un replanteamiento en la ubicación de los servicios.

La captación para las actividades de educación maternal se realiza desde la consulta prenatal con la inscripción de las embarazadas en un libro de registro, anotándose la fecha cronológica de embarazo y citándolas para el comienzo de la primera sesión de actividades. La edad gestacional de comienzo es aproximadamente a los cinco meses de embarazo. Cuando se puso en marcha por primera vez este programa en Jaén en 1986-87 la edad gestacional de inicio era alrededor de los siete meses. El adelanto de la fecha de inicio obedece, según el matrón, a una mayor adecuación de la información proporcionada al tiempo de gestación de la mujer, hay una mayor asunción de conocimientos y una mayor asimilación de los mismos, también al cambio en el enfoque de estas actividades mas centrado ahora en la maternidad y antes en el momento del parto. Otra justificación que nos dio el matrón fue la de dar a la mujer una mayor oferta en el tiempo y flexibilidad para asistir a las diferentes sesiones.

\section{El primer día de las sesiones teóricas.}

El primer día que el matrón cita a las embarazadas para las actividades de educación maternal, lo hace en el centro de salud y las ubica en el único espacio amplio y disponible para tal fin, un vestíbulo que sirve de sala de espera para las consultas y actividades sanitarias que se desarrollan en el centro de salud en otros horarios y días, por lo que este vestíbulo tiene un mobiliario móvil para poder adaptarlo a cada circunstancia -figura 1-.

En la primera sesión teórica el matrón se presenta y en este caso nos dio la palabra para que explicáramos cual era el objeto de nuestra presencia allí. A continuación dio la palabra a las embarazadas para que se presentaran. Estas presentaciones, según nos contó luego, son protocolarias para distender el ambiente que cualquier grupo tiene al interactuar por primera vez. A continuación explica la estructura y contenidos del programa, comienza diciendo que el programa se divide en sesiones teóricas y prácticas y que a su vez las sesiones teóricas tienen una fecha y horario y las sesiones prácticas tienen otra fecha y horario distinto, haciéndole hincapié a las embarazadas para que tomen nota de los horarios, para lo que les proporciona papel y bolígrafo. Desarrolla brevemente los contenidos de cada una de las sesiones teóricas que a lo largo del programa se irán viendo y discutiendo. Así mismo explica de forma más detallada como se estructuran las sesiones prácticas y el lugar de realización. Esta parte práctica se divide a su vez en ejercicios gimnásticos terrestres, ejercicios respiratorios y ejercicios acuáticos. Hace hincapié en la necesidad de entrenar en la casa a diario sobre lo aprendido en las sesiones y advier- 
te de las contraindicaciones generales acerca de la realización de los ejercicios tanto acuáticos como terrestres.

El resto del tiempo de esta sesión inicial el matrón lo utiliza para que las embarazadas multíparas cuenten sus experiencias en embarazos anteriores, en un intento de que el grupo transmita experiencias y sirvan las embarazadas multíparas de referencia a las embarazadas primíparas. Se establece en esta primera sesión una relación similar a la de los grupos etarios pero aquí, el criterio no es la edad cronológica sino edad social basada en la experiencia vivida, liderando el espacio y tiempo las embarazadas "veteranas" a tenor del uso de la palabra. Entendemos ésta una relación de dominación propiciada de forma intencionada por el matrón para que las embarazadas primíparas tomen posición en el grupo y adquieran las primeras referencias tutorizadas de iguales acerca de sensaciones y experiencias del embarazo. Obsérvese en la figura 1 la disposición de las sillas en forma de herradura y la proximidad entre las embarazadas, esto refuerza la solidaridad del grupo y la cohesión entre las embarazadas. En todas las sesiones excepto en la sexta, las embarazadas acuden sin su pareja siendo la proximidad aún mayor al haber menos personas.

\section{Desarrollo de sesiones teóricas siguientes.}

Como hemos puesto de manifiesto nos fue imposible en nuestra investigación hacer un seguimiento completo del programa pero hicimos un esfuerzo por asistir como observadores de nuevo a la segunda sesión teórica de educación maternal. Empieza esta sesión con la cumplimentación de la ficha de educación maternal, advertimos que no están las 15 embarazadas que iniciaron el grupo en la sesión anterior, sí se encontraban las cinco nulíparas. Queda patente en varios estudios realizados que la multiparidad constituye un factor que disminuye la asistencia a los cursos de educación maternal por tener ya la embarazada experiencias previas y menor disponibilidad de tiempo ( Goberna, 1997).

El guión oficial de estas actividades preveía una sesión sobre contenidos de anatomía y fisiología pero la sesión se dirigió por otros derroteros, y esto fue así por que una de las embarazadas había estado ingresada durante 24 horas en el centro materno infantil al advertir unas molestias en el abdomen bajo. La misma embarazada dijo: " no sé si eran contracciones esas molestias por lo que decide ir al maternal, después me pusieron el monitor y me dijeron que no tenía contracciones". Esta situación inesperada hizo cambiar al matrón el contenido que inicialmente tenía preparado para esta segunda sesión. Sobre lo explicado por el matrón quienes más preguntaron fueron las embarazadas primigestas, propio por otra parte, por la novedad de estas sensaciones corporales y en un intento también de ir tomando posiciones en el grupo. A estas alturas de la sesión se nota un ambiente más relajado que en la sesión anterior.

Hecha esta explicación el matrón sigue con la cumplimentación de la ficha y respondiendo a cuantas dudas se planteaban al respecto de lo que en ella decía. Se generó incertidumbre entre las embarazadas acerca de su correcta vacunación del toxoide tetánico a lo que el matrón fue respondiendo de forma individualizada y personalizada. El registro de la parte dedicada a hábitos tóxicos brindó al matrón la oportunidad de hablar sobre estilos de vida saludables y de los peligros del consumo de alcohol, tabaco, medicamentos y otras drogas que pueden resultar teratógenas (Burroug, 1999).

Apuntamos después de lo observado en estas dos sesiones y corroborado por otras dos más a las que asistimos, que la organización y rigidez de las

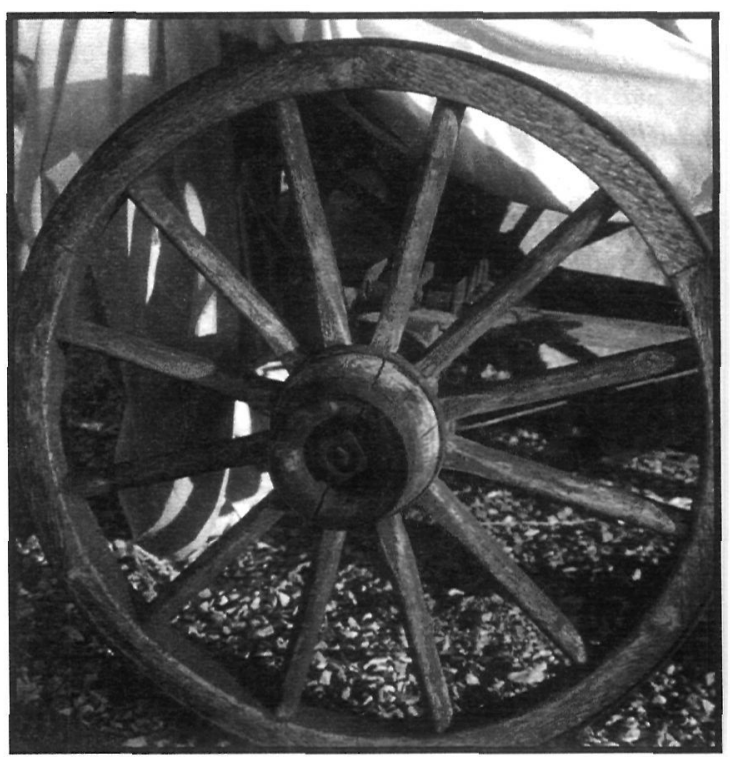


charlas se rompe a favor de las necesidades de la embarazada y en función de las circunstancias que se vayan dando en el grupo. Se trata por tanto de unas sesiones teóricas más flexibles y menos estructuradas que las de años atrás, sintiéndose la embarazada más identificada con el programa, esto a su vez genera multitud de experiencias que amplifican el objetivo de estas sesiones -que la embarazada tenga una experiencia positiva y saludable en su proceso de maternidad-. Justamente al término de esta sesión que fue algo más larga de lo habitual, el matrón nos comentaba al respecto: “El encorsetamiento de las sesiones tradicionales ya no se estila, ahora lo mismo hablas un día de política sanitaria y las mujeres participan de la charla, que hablas de las ventajas de la levadura de cerveza en el embarazo", "Ellas se sienten más identificadas e intervienen más".

A la tercera sesión teórica también asistimos, el matrón nos comenta antes de iniciar la sesión que el tema versará sobre anatomía y fisiología del aparato genital femenino pero centrado en el tema en la lactancia materna puesto que es un objetivo prioritario del Distrito Sanitario fomentar la lactancia materna a través de una sesión específica. Una vez más las preguntas suscitadas por el tema hacen que la sesión se convierta en un intercambio de impresiones focalizadas en la relación entre genitales externos y episiotomía -Incisión quirúrgica que se practica para ampliar el introito vulvar en la fase de la expulsión fetal-. Las preguntas realizadas al matrón al respecto de este tema nos hacen pensar que las embarazadas tienen un temor y desconocimiento bastante grande. Comentarios como: " Dicen que te cortan a lo vivo y que no te ponen anestesia", o " a mí me dieron veintitantos puntos para coserme", denotan falta de información por un lado y alardeo de algunas embarazadas al haber experimentado una práctica desagradable pero que se incluye en la simbología de un rito de paso como es el momento del parto.

El tema sobre la episiotomía deriva en otro tema no programado en la sesión del día como es el de la anestesia epidural y el acompañamiento de la pareja en el parto, dos cuestiones de reciente oferta entre los servicios del sistema sanitario andaluz y reivindicados socialmente desde hace bastante tiempo. Una especie ésta, de relación clientelar entre políticos y ciudadanos en la que a cambio de una oferta sanitaria se asegura el poder en la arena política.

Las dudas que plantea el tema de la anestesia epidural están centradas sobre el derecho de la mujer embarazada a disponer de ella en todos los casos. El matrón una vez más justifica las decisiones de los profesionales, unas veces por la falta de recursos humanos y materiales y otras por las contraindicaciones de la anestesia. Dice: "No os olvidéis que estamos en Jaén" haciendo clara alusión a las limitaciones de los recursos. De esta forma el matrón desde esta posición se convierte en guardián del orden sanitario establecido procurando que la embarazada tenga una actitud de comprensión hacia los profesionales que le van a asistir en el momento del parto.

Acaba la tercera sesión sin tiempo material para abordar el tema de la lactancia materna a lo que el matrón responsable me refiere que lo desarrollará en la quinta sesión teórica. Nos dice que la desmitificación del dolor como eje del parto es un tema que ha dejado de desarrollarse de forma específica y que sobre él se dan mensajes subliminales a lo largo de todo el programa como hemos podido comprobar hoy.

La cuarta sesión se centro en los cuidados generales del recién nacido, más que sobre la diferenciación entre contracciones y movimientos fetales tal y como se venía haciendo tradicionalmente. Esto es una prueba más de la flexibilidad de estas sesiones, siempre teniendo como punto de referencia las demandas del grupo. A esta sesión no pudimos asistir como investigadores pero fuimos informados de sus contenidos cuando asistimos a la sexta sesión.

A la última sesión teórica que asistimos como observadores fue a la sexta y también última de las programadas. El matrón nos comenta antes de iniciar la sesión que el tema de la misma versará sobre la diferenciación entre contracciones de embarazo y parto, signos que acontecen alrededor del día del parto. Esta sesión tiene de especial que asisten las parejas de las mujeres embarazadas a las que da la bienvenida y les comenta el por qué de lo interesante de su asistencia a la sesión. El que los maridos en esta sesión acompañen a las embarazadas viene justificado por dos motivos: Por un lado, 


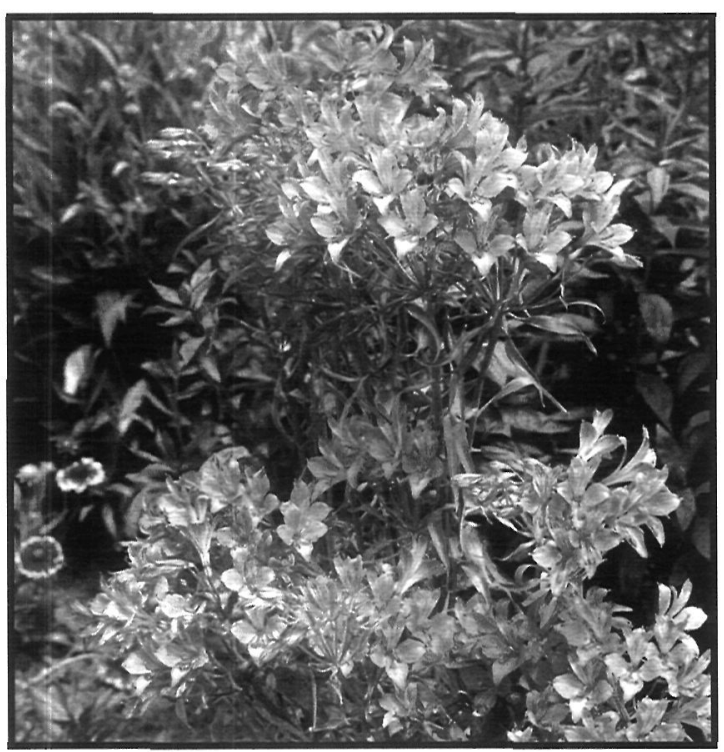

al hablar en esta charla sobre contracciones del embarazo y parto y signos prodrómicos del parto, el responsable cree conveniente que sean ambos los que tengan claras las ideas ante la situación de parto, nos comento: "De nada sirve instruir a la embarazada y que ella tenga las cosas muy claras el día del parto, si el marido no las tiene y titubea ante signos que desconoce" . Por otro lado la cada vez mayor demanda de parejas que quieren acompañar a sus mujeres en el momento del parto ha hecho que en esta sesión se haga un recorrido por los sitios que pasa una mujer desde que ingresa en el centro materno-infantil hasta que es dada de alta después de haber tenido su hijo. Se refuerza esta información con algunas pautas de comportamiento que ha de tener el marido cuando acompaña a su pareja en la sala de dilatación y paritorio.

Nuevamente observamos con estas pautas educativas un intento de mantener el orden social establecido en los paritorios que por otro lado han sido un territorio tabú durante muchos años. La justificación de estas instrucciones acerca del comportamiento al acompañar a la embarazada está en la colaboración de los usuarios de la sanidad para garantizar un trabajo exitoso de los profesionales de la salud, a la vez que se pretende que sean los cónyuges los verdaderos protagonistas de este momento. Esto último puede interpretarse como un intento de devolver el protagonismo del nacimien- to al núcleo familiar de donde nunca debió haber salido, como ocurrió con la asistencia maternal muy medicalizada de las décadas de los años 60 , 70 y buena parte de los 80 . A este cambio de orientación en los paritorios ha contribuido el enfoque natural que recubre ahora al proceso de maternidad y nacimiento, desterrando la idea de enfermedad asociada al embarazo que se ha mantenido durante mucho tiempo.

El tema desarrollado por el matrón en esta sesión despierta una atención muy llamativa entre las parejas, especialmente entre los futuros padres y madres noveles. Muestras de esta especial atención son la mirada fija e invariable al matrón que habla, asentimiento con la cabeza a lo comentado, esbozo de sonrisas y sobre todo las preguntas que realizan sobre cuando acudir al centro maternal. Esta actitud de escucha y atención puede observarse en la misma.

La incorporación del futuro padre a estas sesiones, su interés por lo que le ocurre a la mujer embarazada y el deseo generalizado por acompañarla en el momento del parto tal como expresaron la mayoría, puede ser considerado como una manifestación por conquistar el rol maternal masculino. Algunos autores ven en esta "participación" masculina un logro del hombre del siglo XX. Rich (1996) dice que "cuando un hombre reconoce su paternidad, es posible que asista al parto". Pero esta tesis es androcéntrica y de corte esencialista. Otros autores lo explican sobre la base de factores asociados a los cambios de la familia de occidente, la familia nuclear, la movilidad geográfica y el distanciamiento entre el matrimonio y la familia de origen (Segalen, 1997).

Hemos realizado hasta aquí la descripción y algunas interpretaciones acerca de las sesiones teóricas del programa de educación maternal. Aunque las sesiones teóricas acaban en esta sexta sesión, en el centro de referencia que hemos estudiado las sesiones prácticas continúan hasta que la mujer alcanza prácticamente el embarazo a término.

\section{CONCLUSIONES.}

Hemos presentado en este artículo una descripción etnográfica de las sesiones teóricas de educación maternal en un centro de salud concreto. Somos conscientes de que las limitaciones del 
estudio pueden no haber alcanzado la dimensión completa del tema planteado aquí. Por ello no se trata de un estudio que haya pretendido hacer inferencias sino mas bien describir e interpretar unas actividades determinadas en una población concreta. Sería interesante continuar este estudio con una muestra más amplia de casos y población puesto que creemos que este tipo de investigaciones contribuyen no sólo a adecuar los contenidos de estas sesiones a las necesidades de la embarazada sino a conocer mejor la cosmovisión de la mujer cuando está embarazada. Igualmente sería interesante estudiar si estos programas están llegando a las mujeres con escasez de recursos y problemas sociales. Las conclusiones mas destacadas que hemos sacado de nuestro estudio son:

$1^{\text {a }}$ - Las actividades de educación maternal del centro de salud de las Fuentezuelas están organizadas bajo un prisma innovador que rompe en gran medida con la Educación Maternal tradicional.

$2^{\mathrm{a}}$ - La flexibilidad de sus sesiones teóricas, el tratamiento individualizado y personalizado que se le da a la mujer embarazada en ellas, junto con la dinámica de grupo que se crea, son aspectos importantes a destacar de este programa.

$3^{\text {a }}$ - Las sesiones teóricas le dan oportunidad a las embarazadas para intercambiar experiencias y potenciar la solidaridad y cohesión del grupo. $4^{\mathrm{a}}$ - El programa de educación maternal resuelve dudas relacionadas con la maternidad y el proceso de nacimiento.

\section{BIBLIOGRAFÍA}

- Burroug, A. (1999) Enfermería maternoinfantil. $7^{a}$ ed. Mc-Graw-Hill. Interamericana. México.

- Goberna Tricas, J. (1997) Educación Maternal. Aceptación por parte de la mujer embarazada. Enfermería Clín. 7( 6), 263-267.

- Horton, P.B.; Hunt, CH.L. (1988) Sociología. $3^{a}$ ed. Mc Graw-Hill. México.

- Reeder, S. ; Martín, L.; Koniak, D. (1995) Enfermería Materno-Infantil. 17 ed. Interamericana. McGraw-Hill. Madrid.

- Rich, A. (1996) Nacemos de mujer. La maternidad como experiencia e institución. Cátedra. Madrid.

- Rufo Romero, A. Fernández Merino, J.C. (1987) Guía para la Educación Maternal. Junta de Andalucía. Consejería de Salud. Sevilla.

- Ruiz Olabuénaga, J. I. (1996) Metodología de la Investigación Cualitativa. Universidad de Deusto. Bilbao.

- Sebastiá Gracia, G. ( 2000) Entrenamiento psicofísico en la gestante. Matronas Prof. 1, 4-13.

- Segalen, M. (1997) Antropología histórica de la familia. Taurus Universitaria . Madrid.

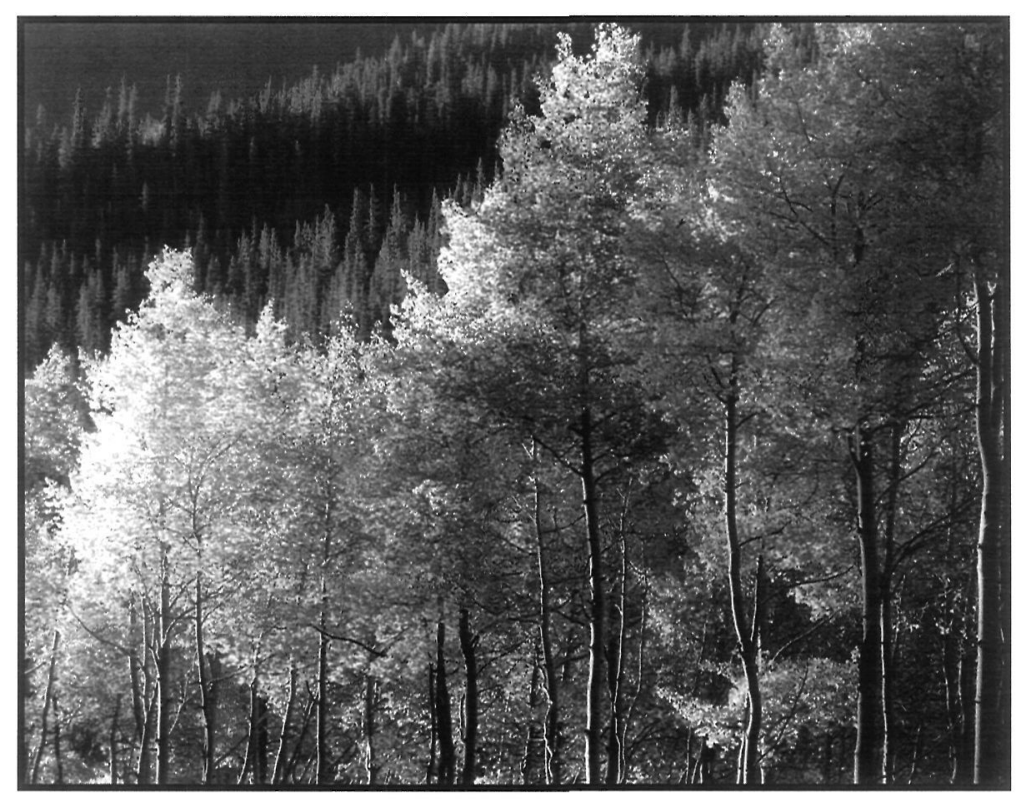

